\title{
O USO DE SENSORES NO ENSINO DE CIÊNCIAS: UMA EXPERIÊNCIA EM AULAS DE FÍSICA
}

\section{THE USE OF SENSORS IN SCIENCE TEACHING: NA EXPERIENCE IN PHYSICAL LESSONS}

\author{
Pedro Miguel Marques da Costa ${ }^{1}$, Marcelo Borges Rocha ${ }^{2}$ \\ ${ }^{1}$ Universidade de Lisboa/Externato Séneca, pedro_mmco@hotmail.com \\ ${ }^{2}$ Centro Federal de Educação Tecnológica Celso Suckow da Fonseca - CEFET/RJ, \\ rochamarcelo36@yahoo.com.br
}

\section{RESUMO}

A tecnologia e a inovação estão cada vez mais presentes no nosso cotidiano e, como tal, também devem ser uma referência na sala de aula. Neste trabalho pretendemos estudar o impacto do uso de sensores e da calculadora gráfica em atividades de Física, no ensino secundário, em Portugal. Foi adotada a metodologia de realização de atividades laboratoriais, recorrendo ao método tradicional e ao uso do sensor e da calculadora gráfica, no sentido de verificar as diferenças na aprendizagem dos alunos, o envolvimento e a motivação em sala de aula. Primeiramente, através de uma avaliação diagnóstica, verificou-se os pré-requisitos essenciais para a realização das atividades laboratoriais e abordagem dos conteúdos, assim como o levantamento das conceções alternativas relacionadas com o tema movimento. No final, os alunos responderam a um questionário sobre o uso da tecnologia e verificou-se que se sentem muito mais motivados e interessados na aprendizagem, que as aulas se tornam diferentes e bem mais interessantes. Sendo assim, infere-se que o uso das Tecnologias de Informação e Comunicação motivam os alunos para a aprendizagem, despertam o interesse e tornam as aulas mais dinâmicas e interativas, promovendo e facilitando o ensino das Ciências.

Palavras-chave: Ensino; Sensores; Calculadora Gráfica; Tecnologia; Física.

\begin{abstract}
Technology and innovation are increasingly present in our daily lives and, as such, should also be a reference in the classroom. In this work we intend to study the impact of the use of sensors and the graphing calculator in Physics activities in secondary education in Portugal. We adopted the methodology of performing laboratory activities, using the traditional method and the use of the sensor and the graphing calculator, in order to verify differences in student learning, involvement and motivation in the classroom. Firstly, through a diagnostic evaluation, the essential prerequisites for carrying out laboratory activities and approach to contents were verified, as well as the survey of alternative concepts related to the movement theme. In the end, the students answered a questionnaire about the use of technology and found that they feel much more motivated and interested in learning, that the classes become different and much more interesting. Thus, it is inferred that the use of Information and Communication Technologies motivate students to learn, arouse interest and make classes more dynamic and interactive, promoting and facilitating the teaching of Science.
\end{abstract}

Key words: Teaching; Sensors; Graphic Calculator; Technology; Physics. 


\title{
INTRODUÇÃOO
}

A sociedade tem sofrido constantes mudanças, muitas destas apoiadas na descoberta de novos conhecimentos. Sendo assim, cabe à escola desenvolver competências nos alunos, para que possam compreender e atuar como cidadãos críticos e responsáveis por suas decisões. Segundo Afonso e Antunes (2001), a educação tem sido apontada como um contributo decisivo (nem sempre problematizado e, por isso, aparentemente consensual) para, nos limites de uma economia globalizada, desenvolver nos indivíduos a percepção das mudanças sociais em curso e as suas consequências. Tudo isso, pautado no conhecimento e nas tecnologias da informação.

Nesse contexto, o papel do professor de Física passa a ser orientar e ajudar os seus alunos no desenvolvimento de conhecimentos científicos e hábitos mentais de que necessitam para se tornarem intelectualmente independentes e capazes de pensar e agir por si mesmos. Não há explicações verbais nem demonstrações suficientemente claras e eficazes que levem à compreensão e ao domínio dos conceitos ou de formas abstratas de raciocínio por parte dos alunos.

\begin{abstract}
"Ser professor é ser um guia, ser um orientador que tem de apoiar os alunos em todos os aspectos. Atribui-se-lhe o papel de facilitador das aprendizagens, o que significa poder ajudar, orientar. Ser professor [...] é fazer com que os alunos sejam competentes para ultrapassar situações, nomeadamente, problemáticas, o que significa formá-los e orientá-los" (Mesquita, 2011, p. 86-87).
\end{abstract}

É essencial envolver a mente dos alunos em pensamento e raciocínio ativos. Segundo Wittgenstein (1953, p. 110) "As pessoas não compreenderão algo apenas porque lhes foi mostrado que é verdadeiro. Temos de situar-nos no seu ponto de vista e fazê-los progredir a partir daí."

“[...] a prática pedagógica tendente a gerar espaços para produzir conhecimento através de diversos métodos, o que quer dizer que é a prática pedagógica o elemento decisivo para fazer de novos modelos e do uso das novas tecnologias propostas inovadoras para a aprendizagem, inovações educativas além de tecnológicas" (Aedo, Garcia e Fadraga, 2001, p.1).

Um dos maiores desafios colocados aos professores está relacionado a integração das Tecnologias de Informação e Comunicação (TIC) na sala de aula, especialmente no ensino das Ciências, e neste caso específico no ensino da Física. O conhecimento científico necessita de uma intervenção planejada por parte do professor, a quem cabe a responsabilidade de sistematizar, tornar simples e claro, sem perder o rigor científico e tendo sempre presente a faixa etária dos alunos, os seus pré-requisitos, os recursos de 
que dispõe e os contextos escolares. De forma implícita ou explícita todos os programas do Ensino Básico e Secundário no âmbito da Física e da Química dão ênfase à utilização das novas tecnologias da comunicação, nomeadamente a utilização de sensores, calculadoras gráficas e computadores.

"Com as tecnologias o ensino torna-se um prazer e serve para educar enquanto professores e alunos estão entretidos. O que envolve o computador, a internet, o chat, email, videoconferência ou qualquer outra utilização tecnológica pode ter um papel importante e colaborar no ensino aprendizagem de forma mais eficaz, envolvente e motivadora." (Moran et al, 2000).

Diante disso, o presente estudo teve o objetivo de investigar as contribuições do uso do sensor de movimento e da calculadora gráfica nas aulas de Física. Sendo assim, foram desenvolvidas quatro atividades numa perspetiva investigativa que inclui uma fase préinterativa de reflexão e discussão e uma fase pós-interativa de análise e crítica de resultados.

\section{O USO DE SENSORES DE MOVIMENTO E DA CALCULADORA GRÁFICA EM SALA DE AULA}

O uso de sensores e da calculadora gráfica, em contexto de sala de aula, é contemplado no programa atual das disciplinas de Física e Química, do Ensino Secundário, para a abordagem e estudo de determinados conteúdos. Na sequência das diretrizes da Direção Geral da Educação Portuguesa (2016), de acordo com os programas em vigor, na disciplina de Física e Química, a utilização de máquinas de calcular gráficas nas aulas deverá ser uma prática habitual em muitas atividades nas quais se utilizam sensores, bem como no tratamento de dados experimentais, incluindo o traçado de gráficos.

Nas atividades experimentais exploradas utiliza-se um sensor de movimento (CBR) e o respectivo software de aquisição e tratamento de dados e uma calculadora gráfica (Fig. 1). Estas atividades podem facilitar a interdisciplinaridade, por exemplo entre a Matemática e a Física, e ajudam alunos e professores a familiarizarem-se com as novas tecnologias. Pretende-se, portanto, que adquiram alguma experiência e confiança na utilização das TIC, sobretudo no uso do CBR e da calculadora gráfica.

Figura 1 - Ligação do gráfica.

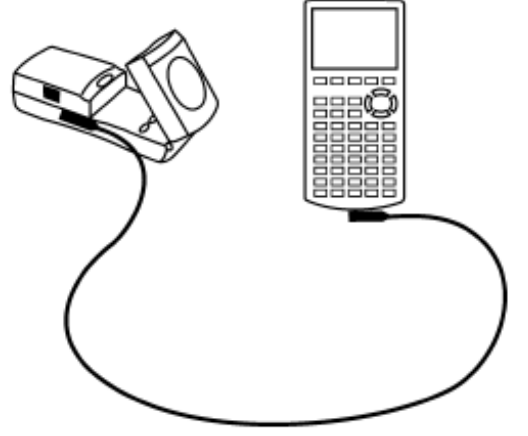


Fonte: https://education.ti.com

A utilização de sensores e de máquinas de calcular na sala de aula pode também resultar numa fonte de motivação, especialmente na resolução de problemas e na realização de atividades experimentais. Permite igualmente uma melhor gestão do tempo de aula, ou seja, com a utilização de sensores diminui-se o tempo despendido durante a aquisição de dados, maximizando o tempo para a análise e discussão dos resultados.

\footnotetext{
"Com algumas características semelhantes às dos computadores, as calculadoras gráficas vieram trazer um novo fôlego à integração da tecnologia no ensino das Ciências. São menos poderosas que os computadores e não os substituem, mas permitem alcançar benefícios educacionais extremamente semelhantes"(Barley, 1994 apud Rocha, 2001, p. 235).
}

A utilização da calculadora gráfica, como instrumento pedagógico, permite aos alunos que, durante a construção dos gráficos avaliem e reavaliem as suas hipóteses possibilitando assim, um método empírico de aprender Física. Neste sentido é aconselhável que os alunos trabalhem em pequenos grupos enquanto que o professor deve prestar o apoio necessário a cada um dos grupos durante a realização das atividades. Assim, os alunos têm oportunidade de debater ideias, formular hipóteses e construir o seu próprio conhecimento num ambiente de experimentação. Morgado e Carvalho (2004, p. 107) salientam a importância dos alunos desenvolverem "competências que lhes permitam continuar a aprender ao longo da vida". Segundo Erduran e Osborne (2005), desenvolver as competências de argumentação, o pensamento crítico, a capacidade reflexiva e de abstração dos alunos torna-se essencial já que têm sido referidas como competências fundamentais numa educação científica que pretenda promover a educação para a cidadania. 
Este tipo de atividade, com o sensor de movimento ou qualquer outro tipo de sensor, com calculadoras gráficas, ou eventualmente com computadores, permitem melhorar o processo de ensino/aprendizagem e, portanto, não podem ser desprezadas pelo professor, quando leciona os conteúdos e define as estratégias de ensino e aprendizagem.

Segundo Sampaio e Simeão (2011), os sensores são cada vez mais utilizados na sociedade atual. $\mathrm{O}$ seu uso no ensino, em atividades práticas, pode potencializar a aprendizagem das ciências e proporcionar um melhor conhecimento e explicação dos fenômenos. Porém é também importante realçar que o uso de sensores (e das novas tecnologias em geral) não deve, contudo, ser encarado como uma receita para todos os problemas que afetam o ensino. Em particular nos níveis mais básicos, continua sendo muito importante desenvolver competências experimentais tais como: planificar e efetuar montagens experimentais; utilizar corretamente instrumentos de medida; construir e analisar tabelas de valores experimentais; elaborar e interpretar gráficos.

"O aluno deduz uma ideia com base em resultados obtidos em atividades laboratoriais/experimentais, ou na análise de informação fornecida ou pesquisada por si (textos, tabelas, esquemas, gráficos, etc.)" (Ministério da Educação, 2013).

\section{O CBR - DEFINIÇÃO E FUNÇÃO}

$\mathrm{O} \mathrm{CBR}^{\mathrm{TM}}$ (Calculator Based Ranger ${ }^{\mathrm{TM}}$ ) é um sensor de movimento ultra-sónico (figura 2), que se liga facilmente a uma calculadora gráfica, através do cabo que o acompanha, permitindo aos alunos recolher, ver e analisar dados de movimento. Assim, podem ser exploradas relações matemáticas e físicas entre distância, velocidade, aceleração e tempo através da recolha e tratamento de dados obtidos nas atividades efetuadas. Podem ser explorados conceitos matemáticos e físicos tais como: movimento - distância, velocidade e aceleração; gráficos - sistemas de eixos, coordenadas, declives e interceções; cálculo - derivadas e integrais; estatística e análise de dados - métodos de recolha de dados e tratamento estatístico. 
Ensino, Saúde e Ambiente-V11 (2), pp.32-54, Agosto. 2018

Figura 2 - Constituição do sensor CBR

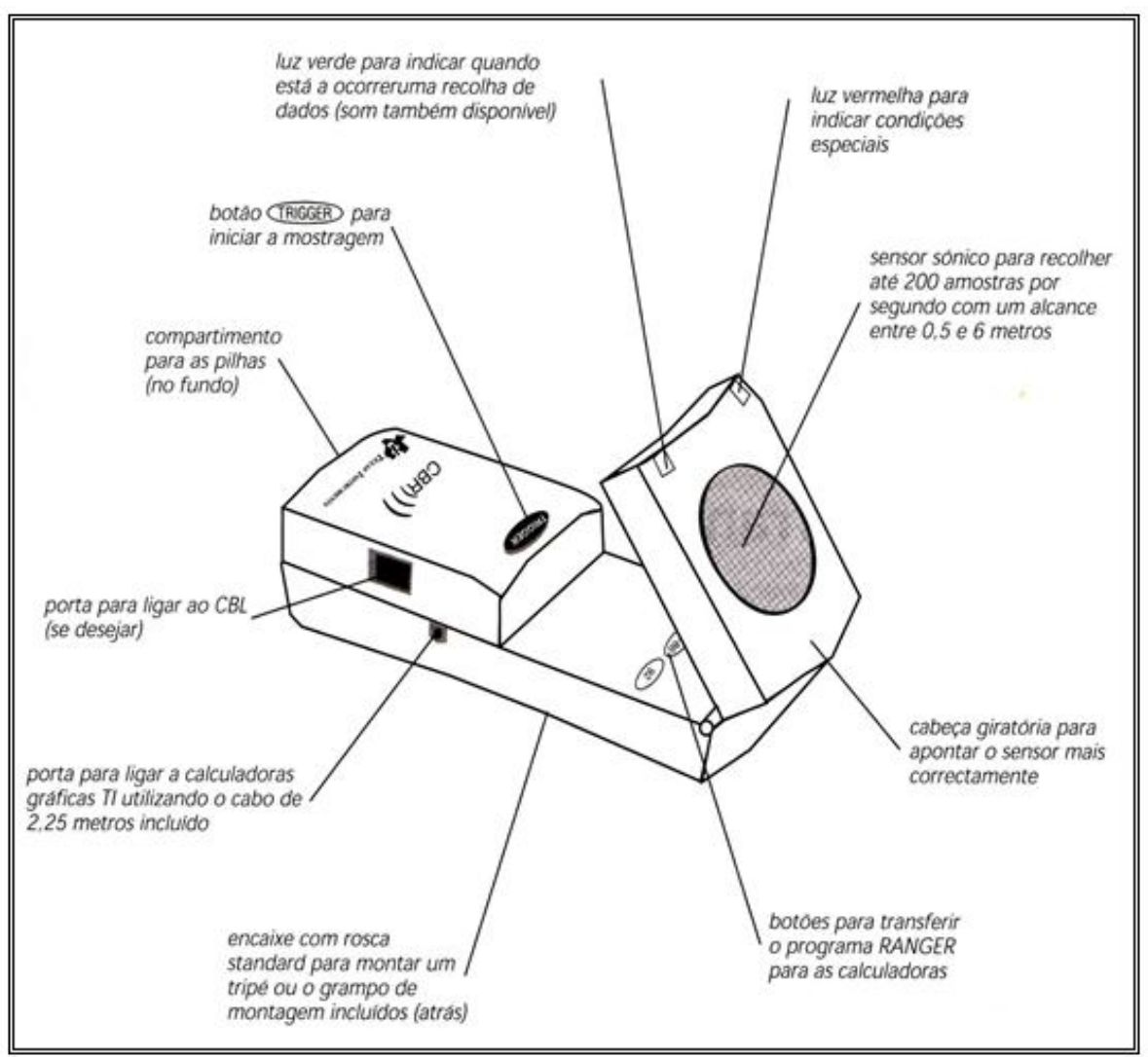

Fonte: https://education.ti.com

Para a coleta de dados, o CBR envia um impulso ultra-sônico e em seguida conta o tempo que esse impulso demora a regressar depois de se ter refletido no objeto mais próximo. Usando este intervalo de tempo e a velocidade de propagação do som, a interface pode determinar a distância a que se encontra o objeto, ou seja, a sua posição em relação ao detetor.

O CBR recolhe dados de distância em função do tempo, envia os dados para uma calculadora gráfica, onde depois são tratados e apresentados graficamente. Em seguida, calcula a primeira e segunda derivadas, dos dados da distância em função do tempo, para obter os valores da velocidade e da aceleração e guarda estes valores nas listas L1, L2, L3 e L4 da calculadora gráfica (Quadro 1).O CBR é fácil de utilizar, autônomo e não necessita de programação. 
Quadro 1 - Dados e grandezas obtidas nas listas da calculadora gráfica.

\begin{tabular}{|c|c|c|c|}
\hline L1 (Lista 1) & L2 (Lista 2) & L3 (Lista 3) & L4 (Lista 4) \\
\hline Tempo & Distância & Velocidade & Aceleração \\
\hline
\end{tabular}

\section{METODOLOGIA}

Com o objetivo de implementar e analisar o uso de sensores e da calculadora gráfica, em atividades laboratoriais de Física do Ensino Secundário, foi selecionada uma turma do $11^{\circ}$ ano de escolaridade, constituída por vinte e sete alunos, de uma escola da região Norte, sub-região do Tâmega, de Portugal, situada em Penafiel, distrito do Porto.

Os alunos desta turma realizaram as atividades laboratoriais recorrendo ao método tradicional e usando o sensor, calculadora gráfica e computador, no sentido de se investigar a motivação, interesse e empenho dos alunos na realização das atividades.

Previamente, durante duas aulas, foi realizado um levantamento dos pré-requisitos necessários e efetuada uma avaliação diagnóstica (Quadro 2) com os alunos. Para esta avaliação diagnóstica, os alunos responderam, individualmente, a um questionário, com questões relacionadas com os conteúdos lecionados, em anos anteriores, na disciplina de física e também matemática.

Quadro 2 - Questionário usado na Avaliação Diagnóstica.

1. Apresente os seguintes valores de grandezas físicas nas unidades indicadas.

A) A massa de uma bola de ténis (57 g) em quilogramas.

B) Duração da primeira parte de um jogo de futebol (45 min) em horas.

C) Distância percorrida por um caracol $(\mathbf{5} \mathbf{~ m m})$ em metros.

D) Potência de um motor $(\mathbf{1 , 3} \mathbf{k W})$ em watts.

E) Energia consumida por uma lâmpada $(25$ W h) em joules.

F) Volume de água numa garrafa $\left(33 \mathbf{~ c m}^{3}\right)$ em metros cúbicos.

G)Corrente elétrica numa pequena lâmpada $(\mathbf{0 , 2 5} \mathbf{~ m A})$ em amperes.

H) Tempo de uma oscilação dos eletrões na rede elétrica $(20$ ms $)$ em segundos.

2. Uma bolacha tem a massa de $10 \mathrm{~g}$. O valor numérico aproximado, em newton, do peso da bolacha é... (Selecione a opção correta)
(A) ... 10
(B) $\ldots 0,10$
(C) .. 0,010
(D) $\ldots 100$

(E)

3. Selecione a opção correta.
(A) $10 \mathrm{~km} / \mathrm{h}=10 \mathrm{~m} / \mathrm{s}$
(B) $10 \mathrm{~km} / \mathrm{h}=10 \times \frac{3600}{1000} \mathrm{~m} / \mathrm{s}$
(C) $10 \mathrm{~m} / \mathrm{s}=10 \times \frac{1000}{3600} \mathrm{~km} / \mathrm{h}$
(D) $10 \mathrm{~km} / \mathrm{h}=10 \times \frac{1000}{3600} \mathrm{~m} / \mathrm{s}$ 
4. O velocímetro digital de um veículo indica $72 \mathrm{~km} / \mathrm{h}$. O valor numérico da velocidade do veículo na unidade SI é... (Selecione a opção correta)
(A) ... 7200
(B) ... 72000
(C)... 20
(D) ... 2

5. Quando um corpo exerce uma força sobre o outro, este exerce também sobre o primeiro uma força de igual módulo e direção, mas de sentido contrário. Esta frase constitui o enunciado da: (Selecione a opção correta)
(A)Lei da Inércia
(B) Lei fundamental da dinâmica
(C)Lei da ação - reação
(D)Lei da conservação da massa

6. Um bloco, como mostra a figura, desloca-se da esquerda para a direita sobre uma superfície horizontal com movimento retardado.

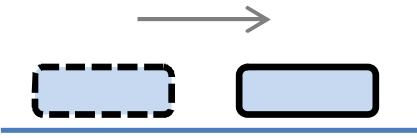

Selecione o diagrama que pode representar as forças que atuam sobre esse bloco.

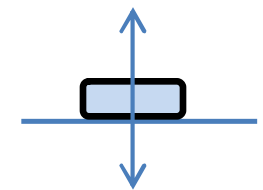

(A)

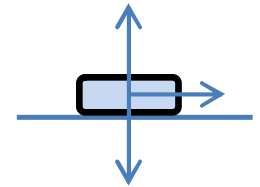

(B)

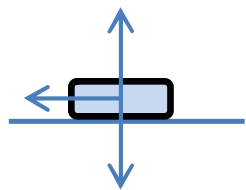

(C)

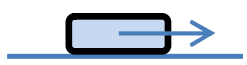

(D)

7. Classifique as afirmações seguintes como verdadeiras (V) ou falsas (F).

(A) A energia cinética é o tipo de energia associada ao movimento.

(B) Quando um corpo sofre a ação de forças cuja resultante não é nula, a sua velocidade varia.

(C) Quando um corpo sofre ação de forças cuja resultante é nula, a sua energia cinética não varia.

(D) A energia cinética de um corpo depende apenas da sua velocidade.

(E) Quanto menor for a velocidade de um corpo, maior é a sua energia cinética.

8. Um bloco é puxado por um rapaz, que exerce uma força de $10 \mathrm{~N}$, como mostra a figura. O bloco move-se retilineamente, sem variação de energia cinética, ao longo de uma superfície horizontal. O atrito não é desprezável.

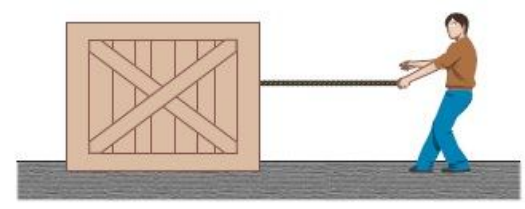

8.1. Marque no esquema as forças aplicadas no bloco. Utilize uma escala adequada.

8.2. Determine a intensidade da força resultante que atua no bloco.

8.3. Calcule a intensidade da força de atrito.

8.4. Selecione a opção que completa corretamente a frase seguinte. "A energia potencial gravítica do sistema bloco + Terra...

(A) ... aumenta, porque o trabalho da força peso é positivo."

(B) ... diminui, porque há atrito."

(C) ... varia, porque a energia cinética é constante."

(D) ... não varia, porque o deslocamento é horizontal." 
9. Considere o gráfico distância-tempo que traduz o movimento de dois automóveis, $\mathbf{X}$ e $\mathbf{Y}$, ao longo de uma trajetória retilínea.

9.1. Indique, justificando, se os automóveis se movem com velocidade constante.

9.2. Indique como se designa o movimento descrito pelos dois automóveis.

10. Considere a seguinte tabela de valores posição-tempo, referente ao movimento

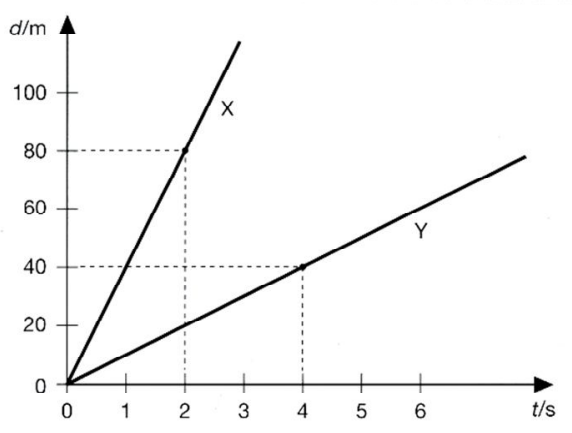
de um motociclista que se desloca numa estrada em linha reta.

\begin{tabular}{|c|c|c|c|c|c|c|}
\hline $\mathbf{t}$ (s) & 0 & 10 & 20 & 30 & 40 & 50 \\
\hline $\mathbf{x}$ (m) & 10 & 30 & 50 & 50 & 70 & 90 \\
\hline
\end{tabular}

10.1. Indique a posição ocupada pelo motociclista no instante $30 \mathrm{~s}$.

10.2. Calcule a velocidade média no intervalo de tempo $[0 ; 10] \mathrm{s}$.

10.3. Indique, justificando, se o motociclista esteve parado durante algum intervalo de tempo.

11. O gráfico velocidade-tempo da figura seguinte traduz o movimento de um motociclo, de $100 \mathrm{~kg}$ de massa, que descreve uma trajetória retilínea.

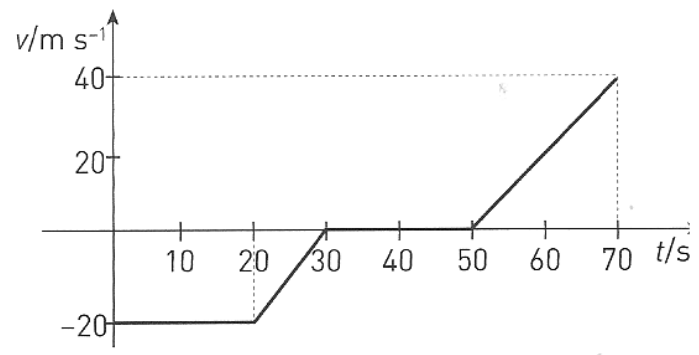

11.1. Classifique o movimento do motociclo nos intervalos de tempo:

$[0 ; 20] \mathrm{s:}$

$[20 ; 30] \mathrm{s:}$

[50;70] s:

11.2. Calcule a distância percorrida nos últimos 20 segundos de movimento.

11.3. Calcule o valor da aceleração média no intervalo de tempo $[20 ; 30] \mathrm{s}$.

11.4. Determine a intensidade da resultante de forças para o intervalo de tempo $[50$; 70] $\mathrm{s}$.

12. Classifique as seguintes afirmações como verdadeiras $(\mathbf{V})$ ou falsas $(\mathbf{F})$ :

(A) Os aparelhos que servem para medir forças chamam-se balanças.

(B) A unidade SI de força é o newton.

(C) Se dois corpos se deslocam com a mesma aceleração, então é garantido que a força resultante neles aplicada tem o mesmo valor.

(D) Um corpo que se move com movimento retilíneo uniforme tem força resultante nula.

(E) Quando a resultante de forças que atuam num corpo é nula, significa que o corpo está parado. 
13. Um jogador chuta uma bola, exercendo uma força de intensidade $60 \mathrm{~N}$.

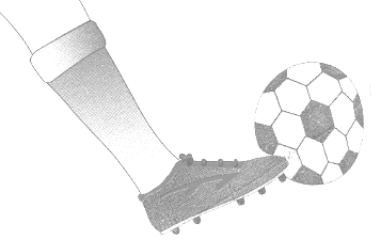

13.1. Represente vetorialmente, na figura, usando uma escala adequada, o par de forças ação - reação.

13.2. Descreva o significado de cada uma das forças representadas.

14. Sabendo que, para completar o seu movimento de translação em torno da Terra, a Lua percorre aproximadamente $2400000 \mathrm{~km}$ em 27 dias e 7 horas, calcule, em $\mathrm{m} / \mathrm{s}$, a rapidez média com que a Lua efetua esse movimento.

Da análise das respostas, dos alunos, foi possível aferir os pré-requisitos existentes e relembrar os que estavam ausentes ou inexistentes. Esta avaliação diagnóstica permitiu, também, trabalhar em sala de aula, estes pré-requisitos, necessários para a realização e exploração, com sucesso, das atividades laboratoriais. Em simultâneo, foram debatidas diversas situações, recorrendo a diferentes recursos, como por exemplo, notícias atuais, que permitiram identificar várias conceções alternativas existentes dado que a mecânica, e o estudo do movimento, um dos temas em que as conceções alternativas se têm demonstrado mais frequentes e difíceis de alterar através do ensino tradicional.

Uma das atitividades realizadas consiste no estudo dos tipos de movimento, nomeadamente o movimento acelerado. Pelo método tradicional, os alunos seguem o protocolo habitual, descrito nos manuais escolares, em que recorrem a materiais de uso comum, como a fita métrica e o cronómetro, que permitem determinar a distância percorrida pelo carrinho e o tempo gasto a percorrer essa distância, fazando variar alguns fatores, como a inclinação da rampa ou a massa do carrinho. Usando o sensor e a calculadora gráfica, foram elaborados, para cada uma das atividades, uma breve introdução teórica (Quadro 3), um protocolo para o professor (Quadro 4) e um protocolo para o aluno (Quadro 5). Os protocolos para o aluno contemplaram, além do procedimento experimental, as previsões para a respetiva atividade, análise da mesma e posterior comparação dos resultados obtidos com as previões previamente efetuadas. 
Quadro 3 - Introdução teórica para a atividade "Movimento Acelerado".

\section{Movimento Acelerado}

A cinemática é o estudo da maneira como os objetos se movem, mas não da razão por que se movem. Galileu estudou muitos aspetos da cinemática, sendo a parte mais valiosa do seu trabalho a que diz respeito aos tipos de movimento. Galileu mostrou, de modo claro e consistente, como se pode descrever o movimento dos objetos com a ajuda de noções matemáticas.

Isaac Newton começou os seus estudos sobre o movimento, na segunda metade do século XVII, na realidade, por Galileu ter sido tão eficaz na descrição do movimento, Newton podia focar a sua atenção sobre a dinâmica, sobre o estudo do porquê de um objeto se mover de uma determinada maneira - porque se começa ele a mover, porque acelera ou se move ao longo de uma trajetória e porque pára.

A cinemática trata da descrição do movimento; por exemplo, ao descrever o movimento de uma pedra deixada cair de um rochedo, poderemos escrever uma equação mostrando como varia a distância percorrida pela pedra em função do tempo decorrido. Poderemos determinar a aceleração e a velocidade alcançadas ao fim de qualquer intervalo de tempo. Mas, ao completar-se a descrição do movimento da pedra, verificaremos que não estamos ainda satisfeitos.

Poderemos perguntar, por exemplo, por que será que a pedra acelera, em vez de se mover com velocidade constante? Porque será que acelera uniformemente? Para responder a estas perguntas teremos que estudar outros conceitos.

A dinâmica vai mais longe do que a cinemática, ao ter em conta as causas do movimento.

Ao estudar cinemática deparámo-nos com três situações; um objeto pode estar em:

(a) Repouso (b) Movimento uniforme (c) Movimento variado (acelerado e retardado)

Para explicar o repouso, o movimento uniforme e o movimento acelerado deveremos ser capazes de responder a perguntas como estas: Porque é que um objeto colocado sobre uma mesa permanece parado? Se der um pequeno impulso a um cubo de gelo em repouso, sobre uma superfície lisa e plana, por que é que ele se move com velocidade uniforme ao longo de uma linha reta, em vez de abrandar rapidamente o seu movimento ou de curvar para um dos lados?

As respostas a estas perguntas e outras perguntas sobre o movimento estão contidas direta ou indiretamente nas três "Leis Gerais do Movimento", formuladas por Isaac Newton. Estas leis surgem no seu famoso livro, Philosophiae Naturalis Principia Mathematica (Princípios Matemáticos da Filosofia Natural, 1687), usualmente referido simplesmente por "Principia".

Nesta atividade pretendemos analisar o movimento de um carrinho que desce uma rampa e depois continua o seu movimento até parar.

Esta atividade experimental enquadra-se no programa de Física $\mathrm{A}-11 .^{\circ}$ ano, na Unidade 1 - Mecânica.

De acordo com a finalidade a que a atividade se destina, pode ser implementada em três ocasiões distintas; a primeira, antes de qualquer exposição dos conteúdos, serve como contexto motivador para os alunos; a segunda ocasião será depois da abordagem de alguns conceitos e a sua finalidade será a sua consolidação e aprofundamento de conceitos; a terceira ocasião será no final da unidade e será uma atividade de sistematização e consolidação dos conteúdos. 
Quadro 4 - Protocolo do Professor para a atividade "Movimento Acelerado".

\section{Protocolo do Professor}

A aceleração média é definida como a variação da velocidade em cada intervalo de tempo. Esta variação poderá ser uma mudança no valor da velocidade, uma mudança de sentido do movimento, ou ambas. Analiticamente, aceleração média é definida como a razão entre a variação de velocidade e o intervalo de tempo em que esta ocorre: $\vec{a}_{m}=\Delta \vec{v} / \Delta t$.

Considera-se que uma partícula está animada de movimento retilíneo uniformemente variado, sempre que se desloque com aceleração média constante, ou seja, quando, a sua velocidade variar do mesmo valor em intervalos de tempo iguais.

Quando a aceleração média e a velocidade média num dado instante apresentarem o mesmo sentido, o movimento diz-se retilíneo uniformemente acelerado, sendo o sentido do movimento positivo para o caso dos valores algébricos da aceleração e da velocidade serem positivos, e negativo para o caso dos valores algébricos da aceleração e da velocidade serem negativos.

Quando a aceleração média e a velocidade média num dado instante têm sentidos opostos, o movimento é retilíneo uniformemente retardado, sendo o sentido do movimento positivo para o caso do valor algébrico da aceleração ser negativo e o da velocidade ser positivo, e negativo para o caso do valor algébrico da aceleração ser positivo e da velocidade ser negativo.

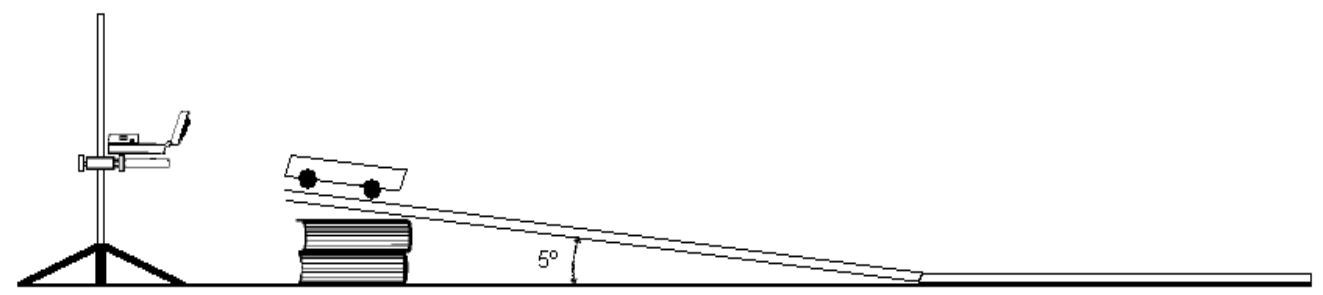

Esquema da montagem

\section{OBJETIVOS}

- Utilizar o sensor de movimento (CBR) para recolher os dados relativos à posição e ao valor da velocidade, à medida que um carro desce uma calha, até que pára.

- Analisar gráficos de posição e velocidade em função de tempo para o movimento acelerado.

- Determinar a expressão matemática que relaciona o valor da velocidade com o tempo para o movimento acelerado.

- Determinar a expressão matemática que relaciona a posição com o tempo para o movimento acelerado.

- Interpretar graficamente as funções $x=f(t), v=f(t), a=f(t)$ para os movimentos retilíneo uniforme e uniformemente variado.

\section{MATERIAL}

Calculadora gráfica TI-83 plus ou superior

CBR (sensor de movimento)

Cabo de ligação

Calha metálica (1,2 a 3 m de comprimento)

Livros (ou outro material para elevar a calha)

Placa de esferovite $(95 \mathrm{~cm} \times 15 \mathrm{~cm} \times 3 \mathrm{~cm})$ 
Suporte universal, garra e noz

TI ViewScreen (opcional)

\section{PROCEDIMENTO}

1. Coloque 2 ou 3 livros a elevar a calha, de modo a que esta forme um ângulo de $5^{\circ}$ com a horizontal. Coloque a esferovite no final da calha de tal modo que a transição da calha para a esferovite seja suave e que carro continue o seu movimento na esferovite até que pare.

2. Fixe o CBR com um suporte universal e com uma garra, a 0,5 $\mathrm{m}$ do topo da calha.

3. Conecte o CBR à calculadora gráfica; empurre firmemente as extremidades do cabo ao efetuar a ligação.

4. Ligue a calculadora e pressione a tecla $\mathbf{0}$, selecione a aplicação CBL/CBR e prima 6. Selecione o programa RANGER. Pressione 6 para iniciar.

5. No MAIN MENU selecione SETUP/SAMPLE para exibir as definições. As definições a utilizar na realização desta atividade deverão ser as seguintes:

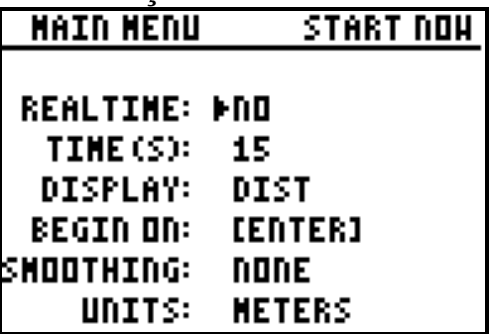

6. Selecione START NOW e pressione a tecla 6.

\section{Parte I - Movimento Acelerado}

7. Segure o carro no topo da calha, tendo especial atenção a que deve estar no mínimo, a $0,5 \mathrm{~m}$ de distância do sensor de movimento.

8. Pressione a tecla 6 para começar a recolher os dados. Depois de começar a ouvir os "cliques" de funcionamento do CBR, segure o carro por mais um segundo e depois solte-o. Retire rapidamente a sua mão do alcance do CBR.

9. Quando a recolha de dados termina é apresentada a representação gráfica da posição em função do tempo.

10. Repita a recolha de dados caso a sua representação gráfica da posição em função do tempo não mostre variações suaves da posição.

11. Para repetir a recolha de dados pressione $\boldsymbol{0}$ e selecione MAIN MENU. Recomece no ponto 6.

12. Avance para a ANÁLISE relativa a esta parte antes de prosseguir para a parte II.

\section{Parte II - Movimento Retardado}

Nesta parte vamos analisar o movimento do carro na esferovite até parar.

13. Repita os procedimentos 7 a 11 efetuados na parte I.

14. Avance para a ANÁLISE relativa a esta parte

\section{ANÁLISE}

\section{Parte I - Movimento Acelerado}

1. O gráfico da posição em função do tempo é apresentado no final da recolha dos dados.

2. Visualize o gráfico velocidade em função do tempo. Para tal, pressione a tecla 6 para voltar ao PLOT MENU. Utilize o cursor para seleccionar VEL-TIME e pressione a tecla $\boldsymbol{6}$. 
3. Determine o valor da aceleração do carro durante o seu movimento na rampa, a partir do gráfico da velocidade. Utilize o cursor da máquina de calcular, no gráfico da velocidade em função do tempo, para obter alguns valores numéricos.

a. Localize no gráfico, o início do movimento acelerado à medida que o carrinho desce a rampa. Registe os valores do instante e da velocidade na tabela referente aos resultados obtidos.

b. Utilize o cursor da calculadora para determinar o momento em que o carro deixou de apresentar movimento acelerado. Registe os valores do instante e da velocidade, na tabela referente aos resultados obtidos.

c. Preencha os restantes campos da tabela.

4. Para analisar com mais pormenor o movimento acelerado, é necessário escolher os pontos que se referem a esse movimento.

a. Pressionar a tecla 6.

b. No PLOT MENU selecionar PLOT TOOLS e pressionar 6.

c. Selecionar SELECT DOMAIN.

d. Utilizar as teclas do cursor, e definir o limite esquerdo no ponto onde o carro inicia o movimento acelerado.

e. Pressionar a tecla $\boldsymbol{6}$ para gravar o limite esquerdo.

f. Utilizar as teclas do cursor, e deslocar o limite direito para o ponto onde o carro termina o movimento acelerado.

g. Pressionar a tecla $\boldsymbol{6}$ para gravar o limite superior.

h. Depois de terminada a seleção dos novos limites do gráfico, a nova seleção do gráfico irá aparecer no ecrã.

5. Quer o gráfico da posição, quer o gráfico da velocidade em função do tempo podem ser ajustados por uma função. No gráfico da velocidade em função do tempo o ajuste deverá ser linear. Com a calculadora é possível ajustar uma função linear aos resultados.

a. Pressione 6 para voltar ao PLOT MENU e depois selecione QUIT.

b. Pressione a tecla e selecione com o cursor o menu CALC.

c. No menu CALC selecione LinearReg $(a x+b)$.

6. Pressione a tecla $\mathbf{0}$, selecione a aplicação CBL/CBR e prima $\boldsymbol{6}$. Selecione o programa RANGER. Pressione $\boldsymbol{6}$ para iniciar.

7. Selecione PLOT MENU e prima ๑. Utilize o cursor para selecionar DISTTIME e pressione a tecla $\boldsymbol{6}$. O gráfico apresentado surge afetado pelos limites escolhidos no ponto 4 .

8. Pressione a tecla 6, selecione QUIT.

9. Em seguida, ajuste uma função quadrática aos dados obtidos para a posição em função do tempo.

a. Pressione a tecla e selecione com o cursor o menu CALC.

b. No menu CALC selecione QuadReg.

10. Retome o ponto 13 do procedimento.

\section{Parte II - Movimento Retardado}

11. Visualize o gráfico velocidade em função do tempo. Para tal, pressione a tecla 6 para voltar ao PLOT MENU. Utilize o cursor para seleccionar VEL-TIME e pressione a tecla $\boldsymbol{6}$.

12. Determine a aceleração do carro durante o seu movimento na esferovite. Utilize as teclas do cursor da máquina de calcular, no gráfico da velocidade em função do tempo, para obter alguns valores numéricos.

a. Localize no gráfico, o início do movimento retardado à medida que o 
carrinho se desloca pela esferovite. Registe os valores do instante e da velocidade na tabela referente aos resultados obtidos.

b. Utilize o cursor da calculadora para determinar o momento em que o carro parou. Registe os valores do instante e da velocidade, na tabela referente aos resultados obtidos.

c. Preencha os restantes campos da tabela.

13. Para analisar com mais pormenor o movimento retardado, é necessário escolher os pontos que traduzem esse movimento.

a. Pressionar a tecla $\boldsymbol{6}$.

b. No PLOT MENU selecionar PLOT TOOLS e pressionar $\boldsymbol{6}$.

c. Selecionar SELECT DOMAIN.

d. Utilizar as teclas do cursor, e definir o limite esquerdo para o ponto onde o carro inicia o movimento acelerado.

e. Pressionar a tecla $\mathbf{6}$ para gravar o limite esquerdo.

f. Utilizar as teclas do cursor, e deslocar o limite direito para o ponto onde o carro termina o movimento acelerado.

g. Pressionar a tecla $\boldsymbol{6}$ para gravar o limite superior.

h. Depois de terminada a seleção dos novos limites do gráfico, a nova seleção do gráfico irá aparecer no ecrã.

14. Ajuste o gráfico da velocidade em função do tempo por uma função linear.

a. Pressione 6 para voltar ao PLOT MENU e depois selecione QUIT.

b. Pressione a tecla e selecione com o cursor o menu CALC.

c. No menu CALC selecione LinearReg $(a x+b)$.

15. Pressione a tecla $\mathbf{0}$, selecione a aplicação CBL/CBR e prima 6 . Selecione o programa RANGER. Pressione 6 para iniciar.

16. Selecione PLOT MENU e prima 6. Utilize o cursor para seleccionar DISTTIME e pressione a tecla $\mathbf{6}$. O gráfico apresentado surge afetado pelos limites escolhidos no ponto 15 .

17. Pressione a tecla $\boldsymbol{\bullet}$, selecione QUIT.

18. Em seguida, ajuste uma função quadrática aos dados obtidos para a posição em função do tempo.

a. Pressione a tecla e selecione com o cursor $\mathrm{o}$ menu CALC.

b. No menu CALC selecione QuadReg.

Quadro 5 - Protocolo do Aluno para a atividade "Movimento Acelerado".

Protocolo do Aluno
Vamos estudar a seguinte situação: um carro que se move no sentido positivo,
ou seja, aumenta a sua distância em relação à origem do referencial. Quando se inicia o
movimento o carro adquire uma aceleração positiva. Posteriormente, quando o carro
diminui o valor da sua velocidade, adquire uma aceleração negativa. Se o carro aumenta
o valor da sua velocidade de forma constante diz-se que o movimento é uniformemente
acelerado.
Nesta atividade, vamos proceder à recolha e análise de dados, referentes à
posição e à velocidade, em duas situações, para um carro que desce uma rampa e depois
continua o seu movimento até parar.




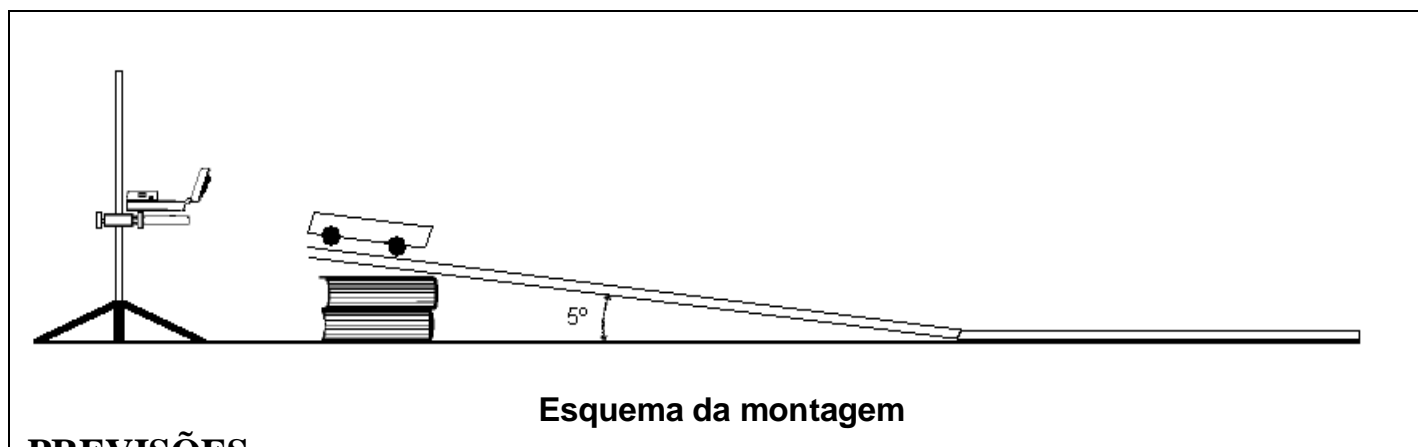

PREVISÕES

1. Esboça um gráfico do valor da velocidade em função do tempo para um carro cuja velocidade aumente uniformemente. Descreve o significado do gráfico por palavras tuas.

2. Esboça um gráfico da posição em função do tempo, para um carro que aumente o valor da sua velocidade uniformemente. Descreve o significado do gráfico por palavras tuas.

3. Esboça o gráfico do valor da velocidade em função de tempo, à medida que o carro diminui a sua velocidade uniformemente. Descreve o significado do gráfico por palavras tuas.

4. Esboça uma representação gráfica da posição em função o tempo, para um carro que diminua a sua velocidade uniformemente, até parar. Descreve o significado do gráfico por palavras tuas.

Responde às seguintes questões, considerando um carro que parte do repouso com movimento retilíneo uniformemente acelerado, durante $10 \mathrm{~s}$.

5. Compara o valor da velocidade do carro após quatro segundos com o valor da velocidade após dois segundos? Qual será o valor da velocidade do carro após dez segundos, quando comparado com o valor da velocidade após dois segundos?

6. Será o espaço percorrido pelo carro maior nos primeiros dois segundos de movimento ou nos últimos dois segundos do movimento? Porquê?

\section{ANÁLISE}

\section{Parte I - Movimento Acelerado}

7. Observa o movimento do carrinho ao longo da calha e depois na esferovite.

8. Esboça o respetivo gráfico da posição em função do tempo. $\mathrm{O}$ gráfico deve apresentar as diferentes regiões que correspondem aos diferentes tipos de movimento em análise. Identifica essas regiões diretamente no esboço do gráfico.

a. Analisa o gráfico da posição em função do tempo e identifica o momento em que o carro estava em repouso no cimo da calha. Regista no gráfico.

b. Identifica o intervalo de tempo durante o qual o carro apresentou movimento retilíneo uniformemente acelerado. Regista no gráfico.

c. Identifica o intervalo de tempo durante o qual o carro apresentou movimento retilíneo uniformemente retardado. Regista no gráfico.

9. O carro ao descer a rampa encontra-se a aumentar ou a diminuir a sua distância em relação à origem? Como justificas tal conclusão?

10. Visualiza o gráfico do valor da velocidade em função do tempo. Esboça o gráfico obtido. $\mathrm{O}$ gráfico apresenta as diferentes regiões que correspondem aos diferentes tipos de movimento. Identifica estas regiões diretamente no esboço do gráfico. 


\begin{tabular}{|c|c|c|c|c|c|}
\hline \multicolumn{6}{|c|}{$\begin{array}{l}\text { a. Analisa o gráfico do valc } \\
\text { o momento em que o ca } \\
\text { no gráfico. } \\
\text { b. Identifica o intervalo } \\
\text { movimento retilíneo unif } \\
\text { c. Identifica o intervalo } \\
\text { movimento retilíneo un } \\
\text { região. } \\
\text { 11. Determina o valor da aceleração } \\
\text { partir do gráfico da velocidade. } \\
\text { gráfico da velocidade em função } \\
\text { a. Localiza no gráfico, o } \\
\text { carrinho desce a rampa. } \\
\text { tabela seguinte. } \\
\text { b. Utiliza o cursor da calo } \\
\text { carro deixou de apresen } \\
\text { instante e da velocidade, } \\
\text { c. Preenche os restantes ca } \\
\text { velocidade }(\Delta v), \text { o corre } \\
\text { aceleração. }\end{array}$} \\
\hline & & & & & \\
\hline \multicolumn{6}{|l|}{ Início do movimento acelerado } \\
\hline \multicolumn{6}{|l|}{ Fim do movimento acelerado } \\
\hline \multicolumn{6}{|c|}{$\begin{array}{l}\text { 12. Para analisar com mais pormenor o m } \\
\text { os pontos, na representação gráfica } \\
\text { traduzem unicamente este movimento } \\
\text { a. Depois de terminada a seleção } \\
\text { gráfico irá aparecer no ecrã. } \\
\text { b. Esboça o gráfico. Descreve o e } \\
\text { 13. Compara o gráfico obtido com as tua } \\
\text { 14. Quer o gráfico do valor da velocidad } \\
\text { tempo podem ser ajustados por uma } \\
\text { em função do tempo o ajuste deverá } \\
\text { ajustar uma função linear aos resultad } \\
\text { a. Anota os valores obtidos para }\end{array}$} \\
\hline \\
\hline \multicolumn{6}{|l|}{ ara a posição $\left(\mathbf{y}=\mathbf{A} \mathbf{x}^{2}+\mathbf{B x}+\right.$} \\
\hline \multicolumn{6}{|c|}{$\begin{array}{l}\text { 15. Compara o coeficiente do termo depend } \\
\text { anterior, com o valor da aceleração ca } \\
\text { concluir? } \\
\text { 16. Qual o significado de cada um dos termo } \\
\text { ajuste linear }(y, A, B) \text {. Com que grandezas } \\
\text { 17. Visualiza o gráfico da posição em fun } \\
\text { apresentado surge afetado pelos limite } \\
\text { representação de } v=f(t) \text { ). Esboça o gráfico } \\
\text { 18. Compara o gráfico obtido com as tuas pre } \\
\text { 19. Em seguida, pode ser ajustada uma função }\end{array}$} \\
\hline
\end{tabular}




\begin{tabular}{l} 
posição em função do tempo. \\
a. Anota os valores obtidos para o ajuste quadrático na tabela de resultados \\
anterior. \\
20. Compara o coeficiente do termo dependente de $x^{2}$, obtido pelo ajuste quadrático \\
dos resultados, com o valor da aceleração calculado no ponto 5? Critica os \\
resultados obtidos. \\
21. Qual o significado de cada um dos termos presentes na equação resultante do \\
ajuste quadrático (y, $A, B$, $C$ )? Com que grandezas físicas se podem relacionar? \\
Parte II - Movimento Retardado \\
22. Visualiza o gráfico do valor da velocidade em função do tempo. \\
23. Determina o valor da aceleração do carro durante o seu movimento na \\
esferovite. Utiliza as teclas do cursor da máquina de calcular, no gráfico do valor \\
da velocidade em função do tempo, para obter alguns valores numéricos. \\
a. Localiza no gráfico, o início do movimento retardado à medida que o \\
carrinho se desloca pela esferovite. Regista os valores do instante e da \\
velocidade na tabela seguinte. \\
b. Utiliza o cursor da calculadora para determinar o momento em que o \\
carro parou. Regista os valores do instante e da velocidade, na tabela \\
seguinte. \\
c. Preenche os restantes campos da tabela. Calcula o valor da variação da \\
velocidade ( $\Delta v)$ o correspondente intervalo de tempo ( $\Delta t)$ e o valor da \\
aceleração. \\
\hline
\end{tabular}

24. Para analisar com mais pormenor o movimento retardado, é necessário escolher os pontos, na representação gráfica do valor da velocidade em função do tempo, que traduzem unicamente este movimento.

a. Depois de terminada a seleção dos limites do gráfico, a nova seleção do gráfico irá aparecer no ecrã.

b. Esboça o gráfico. Descreve o gráfico por palavras próprias.

25. Compara o gráfico obtido com as tuas previsões. O que podes concluir?

26. Ajustar o gráfico da velocidade em função do tempo por uma função linear.

a. Anotar os valores obtidos para o ajuste linear na tabela seguinte.

Ajuste linear dos resultados obtidos para a

velocidade $(\mathbf{y}=\mathbf{A x}+\mathbf{B})$

Ajuste quadrático dos resultados obtidos

para a posição $\left(\mathbf{y}=\mathbf{A x} \mathbf{x}^{2}+\mathbf{B x}+\mathbf{C}\right)$

27. Compara o coeficiente do termo dependente de $x$, obtido no ajuste linear, com o valor da aceleração calculado no ponto 23 ?

28. Visualiza o gráfico da posição em função do tempo. Este gráfico agora apresentado surge afectado pelos limites definidos anteriormente (para a representação de $v=f(t)$ ). Esboça o gráfico obtido.

29. Compara o gráfico obtido com as tuas previsões. O que podes concluir?

30. Em seguida, pode ser ajustada uma função quadrática aos dados obtidos para a posição em função do tempo.

a. Anota os valores obtidos para o ajuste quadrático na tabela de resultados anterior.

31. Compara o coeficiente do termo dependente de $x^{2}$, obtido pelo ajuste quadrático 
dos resultados, com o valor da aceleração calculado no ponto 23? Critica os resultados obtidos?

32. Compara o valor obtido para a aceleração na Parte I e na Parte II. Em que situação foi superior? Que conclusão se pode tirar?

33. O movimento anteriormente estudado pressupõe que o carrinho se move no sentido positivo aumentando a sua distância em relação à origem do nosso referencial (o CBR). Supõe agora que o movimento ao longo da rampa corresponde a uma diminuição da distância à origem $(1,2 \mathrm{~m}, 0,8 \mathrm{~m}, 0,6 \mathrm{~m}, \ldots 0$ m) do referencial.

a. Como seriam afetados os sinais algébricos da velocidade e da aceleração?

b. Para se obterem os mesmos resultados, poderia colocar-se o CBR na outra extremidade?

34. Prevê se o valor da aceleração do carrinho ao longo da rampa será alterado quando se adiciona uma massa ao carrinho. Justifica.

No final da realização das atividades, que decorreram durante oito aulas, foi recolhida a opinião dos alunos, através da aplicação de um questionário individual, sobre o uso do sensor e da calculadora gráfica, na realização das atividades laboratoriais de Física e as principais diferenças, vantagens e desvantagens na realização das atividades, pelo método tradicional e com recurso ao sensor e calculadora gráfica. Para análise dos dados coletados foi utilizada a Análise de Conteúdo, por ser considerada, para este estudo, a mais adequada para uma pesquisa qualitativa que busca de forma interpretativa validar os dados coletados (BARDIN, 2009).

\section{RESULTADOS E DISCUSSÃO}

Para a implementação das atividades experimentais é necessário que os alunos possuam os pré-requisitos que se encontram no quadro 6. A avaliação diagnóstica realizada, previamente, permitiu aferir quais os pré-requisitos adquiridos pelos alunos e os que estavam ausentes e/ou não relembrados.

Quadro 6 - Pré-Requisitos necessários, por disciplina e ano.

\begin{tabular}{|c|l|}
\hline Disciplina & \multicolumn{1}{c|}{ Pré-Requisitos } \\
\hline & - Estabelecer relações de proporcionalidade; \\
Matemática & - Converter unidades de espaço e de tempo; \\
& - Realizar cálculos utilizando equações do $1 .^{\circ}$ e do $2 .^{\circ}$ grau; \\
& - Construir, interpretar e analisar gráficos a duas dimensões. \\
\hline & Conceito de: \\
Física & - Distância e unidades de distância; \\
$\left(7 .^{\circ}\right.$ Ano $)$ & - Tempo e unidades de tempo; \\
& - Rapidez média; \\
\hline Física & - Trajetória. \\
\hline
\end{tabular}


Ensino, Saúde e Ambiente-V11 (2), pp.32-54, Agosto. 2018

\begin{tabular}{|c|c|}
\hline (8..$^{\circ}$ Ano $)$ & - Velocidade média. \\
\hline $\begin{array}{c}\text { Física } \\
\left(9 .^{\circ} \text { Ano }\right)\end{array}$ & $\begin{array}{l}\text { Conceito de: } \\
\text { - Repouso e movimento como conceitos relativos; } \\
\text { - Referencial; } \\
\text { - Posição; } \\
\text { - Instante; } \\
\text { - Intervalo de tempo; } \\
\text { - Espaço percorrido; } \\
\text { - Rapidez média; } \\
\text { - Velocidade média; } \\
\text { - Velocidade instantânea; } \\
\text { - Aceleração média; } \\
\text { - Gráfico posição em função do tempo; } \\
\text { - Gráfico velocidade em função do tempo; } \\
\text { - Cálculo de velocidades médias e acelerações médias. }\end{array}$ \\
\hline $\begin{array}{c}\text { Física } \\
\left(10 .^{\circ} \text { Ano }\right)\end{array}$ & $\begin{array}{l}\text { Conceito de: } \\
\text { - Energia cinética e energia potencial gravítica; } \\
\text { - Lei da conservação da energia. }\end{array}$ \\
\hline $\begin{array}{c}\text { Física } \\
\left(11^{\circ} \text { Ano }\right)\end{array}$ & $\begin{array}{l}\text { Conceito de: } \\
\text { - Posição: coordenadas geográficas e cartesianas; } \\
\text { - Instante; } \\
\text { - Intervalo de tempo; } \\
\text { - Trajetória; } \\
\text { - Espaço percorrido; } \\
\text { - Deslocamento; } \\
\text { - Rapidez média; } \\
\text { - Velocidade média; } \\
\text { - Velocidade instantânea; } \\
\text { - Aceleração média; } \\
\text { - Gráfico posição em função do tempo; } \\
\text { - Gráfico velocidade em função do tempo; } \\
\text { - Movimento retilíneo uniforme e uniformemente variado; } \\
\text { - Altura; } \\
\text { - Força gravitacional e força de atrito; } \\
\text { - 2. }{ }^{2} \text { Lei de Newton. }\end{array}$ \\
\hline
\end{tabular}

No que diz respeito a realização das atividades laboratoriais, com recurso ao sensor e à calculadora gráfica, verificamos, sem dúvida, melhorias na aprendizagem e na motivação dos alunos, quer no envolvimento quer na realização das atividades, e uma mais valia na utilização da tecnologia, em contexto de sala de aula. Estas ilações são corroboradas por outros estudos semelhantes. Segundo Torres, Coutinho e Fernandes (2008), os resultados obtidos tornam claro que a utilização da calculadora gráfica e dos sensores foi determinante na abordagem e exploração das atividades. Os alunos usaram as potencialidades dos sensores para obterem dados resultantes de experiências por eles realizadas, bem como usaram os recursos gráficos da calculadora gráfica e tiraram 
partido das traduções entre tabelas, gráficos e fórmulas. Os alunos partilham desta opinião, pois referem "é muito mais motivante aprender com recurso à inovação e tecnologia, a calculadora gráfica tem muitas potencialidades."

É notório, também, que a utilização da tecnologia, como o sensor e a calculadora gráfica, torna as aulas mais dinâmicas, interativas e motivadoras, facilitando ainda a aprendizagem dos alunos, pois os alunos salientam que "as aulas se tornam bem mais dinâmicas e motivadoras, afinal a Física pode ser aprendida de várias formas"; "aprender com a tecnologia é bem mais interessante, tudo parece diferente, as aulas bem diferentes, prefiro aprender assim”. Os alunos sentem-se muito mais entusiasmados e motivados para a aprendizagem "acho mais interessante e motivante as atividades com a calculadora e com sensores"; "gostei muito mais de usar o sensor e a calculadora gráfica, é bem diferente do que costumamos fazer".

Segundo Werner da Rosa e Trentin (2016), a inserção de sensores pode mostrar-se como uma atração para a realização das atividades, assim como para o desenvolvimento da curiosidade dos estudantes no que diz respeito ao funcionamento dessas tecnologias. O uso dos sensores, na realização das atividades laboratoriais, pode atuar como um instigador da curiosidade dos alunos e servir como fomento à busca por pesquisar e discutir ciência. Além disso, o uso de tecnologias, ainda que de forma demonstrativa, remete o aluno a uma aproximação da escola com as situações vivenciais e cotidianas.

O uso da tecnologia, em sala de aula e na área das Ciências, é uma boa forma de trabalhar as conceções alternativas que os alunos possuem. As conceções que os alunos trazem para a aprendizagem da mecânica são bastante sólidas e difíceis de alterar. Vários escritores têm afirmado que é mais difícil mudar as ideias dos alunos nesta área de introdução à mecânica que em qualquer outra área da ciência. Martins (2006); Tenreiro-Vieira e Magalhãe (2006) e Pires (2009) consideram que uma boa forma de familiarizar os estudantescom os conceitos científicos é aproximando-se a aprendizagem da Ciência dos seus interesses e das suas vivências, numa abordagem da Ciência feita em contexto do mundo real.

O uso de sensores, em comparação com o método tradicional, permite uma abordagem mais ampla dos conteúdos, assim como uma exploração mais eficiente dos resultados e sua respetiva análise. Os gráficos podem ser obtidos e interpretados diretamente, no ecrã da calculadora gráfica, e os alunos podem tirar conclusões no imediato e comparar com as suas previsões. 


\section{CONCLUSÃO}

A partr da análise das respostas dos alunos, percebe-se que a motivação e o interesse dos alunos para a aprendizagem com o uso da tecnologia, neste caso em específico, o sensor e a calculadora gráfica aumentou consideravelmente. Todos consideraram que as aulas se tornaram mais dinâmicas, interativas e despertaram um maior interesse e envolvimento nas atividades propostas na aula de Física.

A era tecnológica, a que os alunos estão habituados no seu cotidiano, não pode ser desprezada, em contexto de sala de aula. As novas tecnologias devem ser um recurso utilizado em estratégias de ensino e aprendizagem por parte do professor. Assim, a utilização de sensores e da calculadora gráfica, em atividades laboratoriais ou outras, são uma importante mais valia para a adesão e envolvimento dos alunos no processo de aprendizagem e aquisição de competências. Estes recursos potencializam a aquisição e o tratamento de dados, em tempo real, e motivam os alunos para a execução das atividades e aprendizagem da Física e outras áreas das Ciências.

Desta forma, o uso de sensores e da calculadora gráfica devem ser encarados como uma excelente e disponível ferramenta, tanto para professores como para alunos, facilitando o processo de ensino e aprendizagem e proporcionando uma aprendizagem ativa, dinâmica e motivadora.

\section{REFERÊNCIAS}

AEDO, R. R. F.; GARCIA, P. M. S. \& FADRAGA, E. C. El aprendizaje con el uso de las nuevas tecnologias de la información e las comunicaciones. Revista

Iberoamericana de Educación, 2001.

AFONSO, A. J. e ANTUNES, F. A redefinição do papel do estado e as políticas educativas. Revista Sociologia, Problemas e Práticas, 37, p. 33-48, 2001.

BARDIN, L. Análise de Conteúdo. São Paulo: Edições 70, 2009.

ERDURAN, S. e OSBORNE, J. Developing arguments. In Alsop, S, Bencze, L. \&Pedretti, E. (Eds). Analysing Exemplary Science Teaching, Maidenhead: Open UniversityPress, p. 106-115, 2005.

FIOLHAIS, C. et al. Metas Curriculares do $3^{\circ}$ ciclo - Ciências Físico-Químicas. Ministério da Educação e Ciência, 2013.

FIOLHAIS, C. et al. Programa de Física e Química A, $\mathbf{1 0}^{\circ}$ e $1^{\circ}$ anos. Ministério da Educação e Ciência, 2014. 
MARTINS. Educação em Ciências e Ensino Experimental, Formação de Professores. Ministério da Educação, 2006.

MESQUITA, E. Competências do Professor, representações sobre a formação e a profissão. Lisboa: Edições Sílabo, 2011.

MORAN J. M.; MASETTO, M. T.; e BEHREN, M. A. Novas tecnologias e mediação pedagógicas. Campinas: Papirus, 2000.

MORGADO, J. C e CARVALHO, A. A. A. Usufruir das Mudanças Curriculares para a Integração das Tecnologias da Informação e Comunicação. Revista de Estudos Curriculares. Ano 2, n. 1, p. 85-120. Braga: Associação Portuguesa de Estudos Curriculares, 2004.

PIRES, D. M. Textos de Apoio não editados. ESEB, Bragança, 2009.

ROCHA, H. Calculadoras gráficas: Que utilização? In Actas do XII Seminário de Investigação em Educação Matemática (p. 233-252). Lisboa: APM, 2001.

SOUSA, A. S.; e SIMEÃO, P. Utilização de sensores no ensino das Ciências. Gazeta de Física. v. 34, n. 3 e 4, p. 1-6, 2011.

TENREIRO-VIEIRA, C.; e MAGALHÃES, S. I. Educação em Ciências para uma articulação Ciência, Tecnologia, Sociedade e Pensamento Crítico: um programa de formação de Professores. Revista Portuguesa da Educação, 19(2). Braga, 2006.

TORRES, T.; COUTINHO, C.; e FERNANDES, J. Aplicações e Modelação Matemática com recurso à calculadora gráfica e sensores. Revista Iberoamericana de Educación Matemática, n. 15, p. 26-31, 2008.

WERNER DA ROSA, C. T.; TRENTIN, M. A. Experimento de condução térmica com e sem uso de sensores e Arduino. Caderno Brasileiro de Ensino de Física. v. 33, n. 1, p. 292-305, 2016.

WITTGENSTEIN, L. Philosophical Investigations. BasilBlackwellLtd, 1953. 